\title{
Uniqueness for continuity equations in Hilbert spaces with weakly differentiable drift
}

\author{
Giuseppe Da Prato • Franco Flandoli • \\ Michael Röckner
}

Received: 18 October 2013 / Published online: 15 May 2014

(C) Springer Science+Business Media New York 2014

\begin{abstract}
We prove uniqueness for continuity equations in Hilbert spaces $H$. The corresponding drift $F$ is assumed to be in a first order Sobolev space with respect to some Gaussian measure. As in previous work on the subject, the proof is based on commutator estimates which are infinite dimensional analogues to the classical ones due to DiPerna-Lions. Our general approach is, however, quite different since, instead of considering renormalized solutions, we prove a dense range condition implying uniqueness. In addition, compared to known results by Ambrosio-Figalli and Fang-Luo, we use a different approximation procedure, based on a more regularizing OrnsteinUhlenbeck semigroup and consider Sobolev spaces of vector fields taking values in $H$ rather than the Cameron-Martin space of the Gaussian measure. This leads to different conditions on the derivative of $F$, which are incompatible with previous work on the subject. Furthermore, we can drop the usual exponential integrability conditions on the Gaussian divergence of $F$, thus improving known uniqueness results in this respect.
\end{abstract}

Keywords Infinite dimensional transport equation $\cdot$ Stochastic calculus of variations · Ornstein Uhlenbeck processes

Mathematics Subject Classification $\quad 35 \mathrm{~F} 05 \cdot 58 \mathrm{D} 20 \cdot 60 \mathrm{H} 07$

\footnotetext{
G. Da Prato $(\varangle)$

Scuola Normale Superiore Pisa, Pisa, Italy

e-mail: daprato@sns.it

F. Flandoli

University of Pisa, Pisa, Italy

e-mail: flandoli@dma.unipi.it

M. Röckner

University of Bielefeld, Bielefeld, Germany

e-mail: roeckner@math.uni-bielefeld.de
} 


\section{Introduction}

Let $H$ be a separable real Hilbert space with inner product $\langle\cdot, \cdot\rangle$ and norm $|\cdot|$. Let $F:[0, \infty) \times H \rightarrow H$ be Borel measurable. In this paper we want to give a new proof for uniqueness of solutions to the corresponding continuity equations informally given as

$$
\frac{d}{d t} \mu_{t}+\operatorname{div}\left(F(t, \cdot) \mu_{t}\right)=0, \quad \mu_{0}=\zeta,
$$

where $\zeta$ is a given initial datum in $\mathscr{P}(H)$, i.e. a probability measure on the Borel $\sigma$-algebra $\mathscr{B}(H)$ of $H$, and the solution $t \mapsto \mu_{t}$ a curve in $\mathscr{P}(H)$. The divergence in (1.1) is meant in the sense of distributions, more precisely one uses the duality between $\mathscr{P}(H)$ and a space of test functions on $[0, \infty) \times H$ which we denote by $\mathscr{D}_{T}$ and which will be specified below. Then one can give (1.1) a rigorous meaning by a weak formulation. More precisely, we fix an orthonormal basis $\left\{e_{n}: n \in \mathbb{N}\right\}$ of $H, T>0$, and set $H_{T}:=[0, T] \times H$. Then we define $\mathscr{D}_{T}$ to be the linear space of all functions $u: H_{T} \rightarrow \mathbb{R}$ such that there exists $N \in \mathbb{N}$ such that

$$
u(t, x)=u_{N}\left(t,\left\langle e_{1}, x\right\rangle, \ldots,\left\langle e_{n}, x\right\rangle\right), \quad x \in H
$$

for some $u_{N} \in C_{b}^{1}\left([0, T] \times \mathbb{R}^{N}\right)$ such that $u_{N}(T)=0$. By $C_{b}^{1}\left([0, T] \times \mathbb{R}^{N}\right)$ we mean the set of all mappings $[0, T] \times \mathbb{R}^{N} \rightarrow \mathbb{R}$ which are continuous and bounded. Then (1.1) can be rigorously written as

$$
\int_{0}^{T} \int_{H} \mathscr{K}_{F} u(s, x) \mu_{s}(d x) d s=-\int_{H} u(0, x) \zeta(d x), \quad \forall u \in \mathscr{D}_{T},
$$

where for $(t, x) \in H_{T}, \mathscr{K}_{F}$ is a (degenerate) Kolmogorov operator defined by

$$
\mathscr{K}_{F} u(t, x)=\frac{\partial}{\partial t} u(t, x)+\langle F(t, x), D u(t, x)\rangle
$$

and $D u(t, x) \in H$ is defined through

$$
\langle D u(t, x), y\rangle=u^{\prime}(t, x)(y), \quad y \in H,
$$

where $u^{\prime}(t, x)(\cdot)$ means first Fréchet derivative of $u(t, \cdot)$ with respect to $x \in H$. We note that $\mathscr{D}_{T}$ depends on the chosen orthonormal basis. But this is irrelevant because what is important about the chosen test functions spaces in regard to uniqueness, is that (1.2) makes sense and that it is as small as possible (to make the uniqueness result as strong as possible). A minimal requirement is that it should separate the points of $H$, which obviously holds for $\mathscr{D}_{T}$ defined above by the Hahn-Banach theorem, which in turn by a monotone class argument implies that $\mathscr{D}_{T}$ is dense in every $L^{p}\left(H_{T}, \boldsymbol{v}\right), p \in$ $[1, \infty)$, for any finite (nonnegative) measure $\boldsymbol{v}$ in $H_{T}$.

The main aim of this paper is to find conditions on $F$ such that (1.2) has at most one solution for a given initial condition $\zeta \in \mathscr{P}(H)$. 
In contrast to the Fokker-Planck equation where $\mathscr{K}_{F}$ in (1.2) and (1.3) has a second order part (in $x$ ), and uniqueness is known even for just measurable $F$ (satisfying some integrability assumption), provided the second order part is non degenerate (cf. [5] and the preprint [6]), for the continuity equation at least weak differentiability of $F$ (or being of bounded variation) is required to hope to have uniqueness of solutions, even in finite dimensions (see [1,9]). However, in order to define weak differentiability of a function one needs a reference measure, and if $H=\mathbb{R}^{n}$, e.g. the Lebesgue measure is a natural choice. If $H$ is infinite dimensional, Lebesgue measure does not exist and we have to choose a reference measure on $H$. There is really no canonical choice, but a "good" choice is to take a non degenerate centered Gaussian measure $\mu$ on $H$, because the concept of weak differentiability (with respect to such a $\mu$ ) has been extensively developed in the past in the framework of the Malliavin calculus $[3,13,14]$. This choice of a reference measure was proposed in [2] and they proved existence and uniqueness of solutions to (1.2) under certain conditions on the weak derivative and exponential $\mu$-integrability conditions on its $\mu$-divergence. (see [2, Theorem 3.1], see also [11] for improvements of the results on the corresponding transport equation in [2]).

In this paper, also taking a Gaussian measure $\mu$ as a reference measure, we prove uniqueness for (1.2) by a completely different method. On the other hand, our assumption on the weak derivative of $F$ is different and, in fact incompatible with that in [2], since we use $H$ instead of the Cameron-Martin space as tangent space when defining Sobolev spaces (see Remark 2.6 below). As a consequence, in contrast to [2] we do not need to assume any exponential $\mu$-integrability conditions on the Gaussian divergence of $F$. The idea of proof is inspired by the uniqueness proof for Fokker-Planck equations in Hilbert spaces from $[4,5]$. More precisely, we prove a suitable rank condition for the Kolmogorov operator in (1.3). But to implement this idea we have to regularize with a much more smoothing Ornstein-Uhlenbeck semi-group than the one in $[2,11]$ (see Sect. 2 below). Crucial is again the commutator estimate, which as turns out, can be proved also for this regularization (see Sect. 3).

Let us remark that here we use the commutator estimate to prove a range condition, opposite to the classical works where the commutator estimate is used to prove renormalization of weak solutions. It is at this point that, in [2] and [11], exponential integrability is necessary; for our range condition we do not need it. Concerning the problem of proving a range condition itself, this is usually done by means of gradient estimates on solutions, which is a difficult problem; here we have the gradient estimate for free, see (2.8), because it holds for the $P_{\epsilon}$-regularized solution.

Choosing a reference measure as in $[2,11]$ we also have to restrict to a sub-class of solutions $\mu_{t}, t \in[0, \infty)$, to (1.2), namely those satisfying

$$
\mu_{t}(d x) d t=\rho(t, x) \mu(d x) d t
$$

for some functions $\rho \in L^{p}\left(H_{T}, d t \otimes \mu\right), p>1$, and prove uniqueness in this class.

It is the subject of our further study to relax this condition (1.4), e.g. by considering more general reference measures than Gaussian measures. First steps in this direction have recently be done in [12], where the Gaussian measure $\mu$ is replaced by a measure $v$ which is differentiable in the sense of Fomin (see [3]). In particular, one can take certain 
Gibbs measures for $v$. However, the techniques in that paper are entirely different from our approach here.

This paper exclusively deals with uniqueness results. For existence we refer to the above mentioned papers [12] and [2], [11]. Concerning the latter two, however, we would like to point out that the overlap of the conditions there and ours is quite small (see Remark 2.6 below for details). The existence of solutions to (1.1) will be analyzed in detail in forthcoming work.

We end this section recalling some results about the Ornstein-Uhlenbeck semigroup $P_{t}$ needed in what follows. First we choose and fix an arbitrary centered, non degenerate, Gaussian measure $\mu$ on $H$. Let $Q$ be its covariance operator. So, $Q$ is symmetric, nonnegative definite with kernel $=\{0\}$ and $\operatorname{Tr} Q<\infty$. We also use the notation $\mu=N_{Q}$. Then $P_{t}$ is, for $\varphi \in B_{b}(H)$, defined as

$$
P_{t} \varphi(x)=\int_{H} \varphi(y) N_{T_{t} x, Q_{t}}(d y), \quad x \in H,
$$

where

$$
T_{t}:=e^{-\frac{t}{2} Q^{-1}}, \quad Q_{t}=Q S_{t}^{2}, \quad S_{t}:=\left(1-T_{t}^{2}\right)^{1 / 2} .
$$

$N_{T_{t} x, Q_{t}}$ denotes the Gaussian measure on $H$ with covariance operator $Q_{t}$ and mean $T_{t} x$ and $B_{b}(H)$ is the space of all real and bounded Borel funcions on $H$. We note for further use that

$$
T_{t}^{2}+S_{t}^{2}=1
$$

Consequently the matrix on $H \times H$

$$
\mathscr{R}:=\left(\begin{array}{cc}
T_{t} & S_{t} \\
-S_{t} & T_{t}
\end{array}\right)
$$

is orthogonal, so that the measure $\mu \times \mu$ on $H \times H$ is invariant for $\mathscr{R}$.

Since $N_{T_{t} x, Q_{t}} \ll N_{Q}$, we can write

$$
P_{t} \varphi(x)=\int_{H} \varphi(y) \rho(t, x, y) \mu(d y)
$$

where

$$
\rho(t, x, y)=K(t) \exp \left\{-\frac{1}{2}\left\langle Q_{t}^{-1} T_{t} x, T_{t} x\right\rangle+\left\langle Q_{t}^{-1} T_{t} x, y\right\rangle-\frac{1}{2}\left(\left\langle Q_{t}^{-1} T_{t} y, T_{t} y\right\rangle\right\},\right.
$$

where $K(t)=\left[\operatorname{det}\left(1-T_{t}^{2}\right)\right]^{-1 / 2}$.

We notice, for further use, the following identities.

$$
\begin{aligned}
& D_{x} \rho(t, x, y)=Q_{t}^{-1} T_{t}\left(y-T_{t} x\right) \rho(t, x, y) \\
& D_{y} \rho(t, x, y)=Q_{t}^{-1} T_{t}\left(x-T_{t} y\right) \rho(t, x, y)
\end{aligned}
$$


We finally recall the Mehler formula

$$
P_{t} \varphi(x)=\int_{H} \varphi\left(T_{t} x+S_{t} y\right) \mu(d y) .
$$

\section{The main result and scheme of the proof}

Definition 2.1 A family $\left(\mu_{t}\right)_{t \in[0, T]}$ is called a solution of the (heuristic) continuity equation (1.1) if $\mu_{t} \in \mathscr{P}(H)$ for every $t \in[0, T], t \mapsto \mu_{t}(A)$ is $\mathscr{B}(H)$-measurable for all $A \in \mathscr{B}(H), F \in L^{1}\left(H_{T}, \mu_{t} d t\right)$ and (1.2) holds.

As mentioned in the introduction we need a reference measure on $H$. So let $\mu=N_{Q}$ be the centred, non degenerate, Gaussian measure on $H$ from the Introduction with covariance operator $Q$. Let $\left\{e_{k}: k \in \mathbb{N}\right\}$ be the eigenbasis of $Q$ and $\lambda_{k} \in(0, \infty)$ the corresponding eigenvalues (i.e. $Q e_{k}=\lambda_{k} e_{k}, k \in \mathbb{N}$ ) numbered in decreasing order. Let the test function space $\mathscr{D}_{T}$ be defined as in the introduction with respect to this orthonormal basis $\left\{e_{k}: k \in \mathbb{N}\right\}$.

Define the following subclass $\mathscr{M}_{F, \zeta, p}$ of solutions to (1.1) for fixed initial condition $\zeta \in \mathscr{P}(H)$ and fixed $p \in[1, \infty] \cdot \mathscr{M}_{F, \zeta, p}$ is defined to be the set of all measures $\boldsymbol{\mu}(d t, d x)=\mu_{t}(d x) d t$ such that $\left(\mu_{t}\right)_{t \in[0, T]}$ is a solution to (1.1) in the sense of Definition 2.1 which satisfy

$$
\mu_{t}(d x) d t=\rho(t, x) \mu(d x) d t, \quad \text { for some } \rho \in L^{p}\left(H_{T}, d t \otimes \mu\right)
$$

Clearly, $\mathscr{M}_{F, \zeta, p}$ is a convex set.

The following result is inspired by $[4,5]$

Proposition 2.2 Suppose the following rank condition holds:

$$
\mathscr{K}_{F}\left(\mathscr{D}_{T}\right) \text { is dense in } L^{p^{\prime}}\left(H_{T}, d t \otimes \mu\right) \text {, }
$$

where $p \in[1, \infty]$ and $p^{\prime}=\frac{p}{p-1}$. Then $\mathscr{M}_{F, \zeta, p}$ contains at most one element.

Proof Let $\mu_{t}^{(i)}(d x) d t=\rho^{(i)}(t, x) \mu(d x) d t, i=1,2$ be two elements in $\mathscr{M}_{F, \zeta, p}$. Then by (1.2)

$$
\int_{0}^{T} \int_{H} \mathscr{K}_{F} u(t, x)\left(\rho^{(2)}(t, x)-\rho^{(1)}(t, x)\right) \mu(d x) d t=0, \quad \forall u \in \mathscr{D}_{T} .
$$

Hence $(\mathscr{R})$ implies $\rho^{(1)}=\rho^{(2)}$.

Let us briefly recall the notion of (some) Sobolev spaces of functions on $H$ with respect to $\mu$. 
Let $\mathscr{F} C_{b}^{1}$ ("finitely based $C_{b}^{1}$ functions") denote the linear space of all functions $\varphi: H \rightarrow \mathbb{R}$ such that for some $N \in \mathbb{N}$

$$
\varphi(x)=\varphi_{N}\left(\left\langle e_{1}, x\right\rangle, \ldots,\left\langle e_{N}, x\right\rangle\right), \quad x \in H,
$$

for some $\varphi_{N} \in C_{b}^{1}\left(\mathbb{R}^{N}\right)$. For $p \in[1, \infty)$ equip $\mathscr{F} C_{b}^{1}$ with the norm

$$
\|\varphi\|_{1, p}:=\left(\int_{H}\left(|D \varphi(x)|^{p}+|\varphi(x)|^{p}\right) \mu(d x)\right)^{1 / p},
$$

where $D \varphi(x)$ is the unique element in $H$ such that

$$
\langle D \varphi(x), y\rangle_{H}=\varphi^{\prime}(x)(y)=\frac{\partial \varphi}{\partial y}(x), \quad y \in H,
$$

where $\frac{\partial \varphi}{\partial y}$ means partial derivative in the direction $y$ and $\varphi^{\prime}$ denotes the Fréchet derivative of $\varphi$. Then it is well-known (see e.g. [8]) that $\|\varphi\|_{1, p}$ is closable over $L^{p}(H, \mu)$ so that

$$
\left.W^{1, p}(H, \mu)={\overline{\mathscr{F}} C_{b}^{1}}^{\|\cdot\|_{1, p}} \text { (= completion of } \mathscr{F} C_{b}^{1} \text { with respect to }\|\cdot\|_{1, p}\right)
$$

is a subspace of $L^{p}(H, \mu)$. Likewise as this Sobolev space of functions one defines Sobolev spaces of vector fields $F: H \rightarrow H$ and even of time dependent vector fields $F: H_{T} \rightarrow H$ as follows: let $\mathscr{V} \mathscr{F} C_{b, T}^{1}$ ("finitely based $C_{b}^{1}$ vector fields") denote the linear space of all maps $F: H_{T} \rightarrow H$ such that for some $N \in \mathbb{N}$

$$
F(t, x)=\sum_{i=1}^{N} g_{i}(t, x) e_{i}, \quad(t, x) \in H_{T},
$$

for some $g_{i}: H_{T} \rightarrow \mathbb{R}$ of type

$$
g_{i}(t, x)=g_{i, N}\left(t,\left\langle e_{1}, x\right\rangle, \ldots,\left\langle e_{N}, x\right\rangle\right), \quad x \in H,
$$

with $g_{i, N} \in C_{b}^{1}\left([0, T] \times \mathbb{R}^{N}\right)$. For $p \in[1, \infty)$ we equip $\mathscr{V} \mathscr{F} C_{b, T}^{1}$ with the norm

$$
\|F\|_{1, p, T}:=\left(\int_{0}^{T} \int_{H}\left(\|D F(t, x)\|_{\mathscr{L}_{2}(H)}^{p}+|F(t, x)|_{H}^{p}\right) \mu(d x) d t\right)^{1 / p}
$$

where $\mathscr{L}_{2}(H)$ denotes the linear space of all Hilbert-Schmidt operators on $H$ with corresponding norm $\|\cdot\|_{\mathscr{L}_{2}(H)}$ and

$$
D F(t, x):=\sum_{i=1}^{N}\left\langle D g_{i}(t, x), \cdot\right\rangle e_{i} \in \mathscr{L}_{2}(H) .
$$


Again, it is well known that this norm is closable in $\left.L^{p}\left(H_{T} ; \mathscr{L}_{2}(H), \mu\right)\right)$. Hence we can define the Sobolev space of time dependent vector fields by

$$
L^{p}\left([0, T] ; W^{1, p}(H ; H, \mu)\right)=\text { completion of } \mathscr{V} \mathscr{F} C_{b, T}^{1} \text { with respect to }\|\cdot\|_{1, p, T},
$$

which by closability is a subspace in $L^{p}\left(H_{T} ; H, \mu\right)$.

Now we can formulate our main result.

Theorem 2.3 Let $p \in(2, \infty)$ and suppose that, for some $s>p^{\prime}=\frac{p}{p-1}$, we have $F \in L^{s}\left([0, T] ; W^{1, s}(H ; H, \mu)\right)$ and that, in addition,

$$
F\left(H_{T}\right) \subset Q^{1 / 2}(H) \text {, and } \int_{0}^{T} \int_{H}\left|Q^{-1 / 2} F(t, x)\right|^{S} \mu(d x) d t<\infty \text {. }
$$

Then the rank condition $(\mathscr{R})$ holds, hence by Proposition $2.2 \mathcal{M}_{F, \zeta, p}$ contains at most one element.

The rest of this section is devoted reducing the proof of $(\mathscr{R})$ and hence of Theorem 2.3 to Proposition 2.4 below, which is a commutator estimate for a suitable regularization through the Mehler type semigroup $P_{t}, t \geq 0$, of integral operators on $B_{b}(H)$ defined in (1.5) (see also (1.13)) Let us define the commutator for $u \in \mathscr{D}_{T}, F \in \mathscr{V} \mathscr{F} C_{b, T}^{1},(t, x) \in H_{T}$

$$
B_{\epsilon}(u, F)(t, x):=\left\langle F(t, x), D P_{\epsilon}(u(t, \cdot))(x)\right\rangle-P_{\epsilon}(\langle F(t, \cdot), D u(t, \cdot)\rangle)(x) .
$$

Proposition 2.4 Let $p \in(2, \infty)$ and $r \in(1, \infty), s \in(1,2]$ such that $\frac{1}{p^{\prime}}=\frac{1}{r}+\frac{1}{s}$. Then:

(i) There exists $C \in(0, \infty)$ (independent of $\epsilon$ ) such that

$$
\begin{aligned}
& \left(\int_{0}^{T} \int_{H}\left|B_{\epsilon}(u, F)\right|^{p^{\prime}} d \mu d t\right)^{1 / p^{\prime}} \\
& \quad \leq C\|u\|_{L^{r}\left(H_{T}, d t \otimes \mu\right)} \quad\left(\|F\|_{1, s, T}+\left\|Q^{-1 / 2} F\right\|_{L^{s}\left(H_{T}, d t \otimes \mu\right)}\right),
\end{aligned}
$$

for all $u \in \mathscr{D}_{T}, F \in \mathscr{V} \mathscr{F} C_{b, T}^{1}$. In particular, $B_{\epsilon}$ extends to a continuous bilinear map (denoted by the same symbol)

$$
\begin{gathered}
B_{\epsilon}: L^{r}\left(H_{T}, d t \otimes \mu\right) \times L^{s}\left([0, T] ; W^{1, s}(H ; H, \mu)\right. \\
\left.\cap L^{s}\left(H ; Q^{1 / 2} H ; \mu\right)\right) \rightarrow L^{p^{\prime}}\left(H_{T}, d t \otimes \mu\right) .
\end{gathered}
$$

(ii) $B_{\epsilon}(u, F) \rightarrow 0$ in $L^{p^{\prime}}\left(H_{T}, d t \otimes \mu\right)$ as $\epsilon \rightarrow 0$, for all

$$
(u, F) \in L^{r}\left(H_{T}, d t \otimes \mu\right) \times L^{s}\left([0, T] ; W^{1, s}(H ; H, \mu) \cap L^{s}\left(H ; Q^{1 / 2} H, \mu\right)\right) .
$$


The proof of Proposition 2.4-(i) is carried out in Sect. 3 below. Assertion (ii) obviously holds for all $u \in \mathscr{D}_{T}, F \in \mathscr{V} \mathscr{F} C_{b, T}^{1}$. But by (i), $B_{\epsilon}, \epsilon \in[0,1]$, are equicontinuous on

$$
L^{r}\left(H_{T}, d t \otimes \mu\right) \times L^{s}\left([0, T] ; W^{1, s}(H ; H, \mu) \cap L^{s}\left(H ; Q^{1 / 2} H, \mu\right)\right),
$$

which contains $\mathscr{D}_{T} \times \mathscr{V} \mathscr{F} C_{b, T}^{1}$ as a dense set. Hence (ii) follows.

Let us now show that Proposition 2.4 implies Theorem 2.3.

Claim 2.5 Proposition 2.4 implies $(\mathscr{R})$.

Proof Let $f \in \mathscr{D}_{T}$ and $r, s \in[1, \infty)$ be as in Proposition 2.4 such that $s \in\left(p^{\prime}, 2\right]$. By definition of $L^{s}\left([0, T] ; W^{1, s}(H ; H, \mu)\right)$ there exists $F_{n} \in \mathscr{V} \mathscr{F} C_{b, T}^{1}, n \in \mathbb{N}$, converging to $F$ w.r.t. $\|\cdot\|_{1, s, T}$ and in the sense of Lemma 4.1 of Appendix 1. Since $F_{n}$ is smooth and finitely based, there exists a solution $u_{n} \in \mathscr{D}_{T}$ of

$$
\left\{\begin{array}{l}
\frac{\partial u_{n}}{\partial t}+\left\langle F_{n}, D u_{n}\right\rangle=f, \\
u_{n}(T, \cdot)=0
\end{array}\right.
$$

We namely set

$$
u_{n}(t, x)=-\int_{t}^{T} f\left(s, \xi_{n}(s, t, x)\right) d s
$$

where the characteristics $\xi_{n}(s, t, x)$ are, as well known, the solution to

$$
\frac{\partial}{\partial s} \xi_{n}(s, t, x)=F_{n}\left(s, \xi_{n}(s, t, x)\right), \quad \xi_{n}(t, t, x)=x .
$$

Applying $P_{\epsilon}$ for $\epsilon>0$ to (2.5) we obtain

$$
\frac{\partial P_{\epsilon} u_{n}}{\partial t}+\left\langle F, D P_{\epsilon} u_{n}\right\rangle=P_{\epsilon} f+\left\langle F-F_{n}, D P_{\epsilon} u_{n}\right\rangle+B_{\epsilon}\left(F_{n}, u_{n}\right),
$$

note that $P_{\epsilon} u_{n} \in \mathscr{D}_{T}$.

By (2.6) we have

$$
\left\|u_{n}\right\|_{\infty} \leq\|f\|_{\infty}, \quad \forall n \in \mathbb{N}
$$

and by well known smoothing properties of $P_{\epsilon}$ (see e. g. [7]) we know that for some $C \in(0, \infty)$

$$
\left\|D P_{\epsilon} u_{n}\right\|_{\infty} \leq C \epsilon^{-1 / 2}\left\|u_{n}\right\|_{\infty} \leq C \epsilon^{-1 / 2}\|f\|_{\infty}, \quad \forall n \in \mathbb{N} .
$$

Hence, passing to a subsequence if necessary, we may assume that $u_{n} \rightarrow u$ in $L^{r}\left(H_{T}, d t \otimes \mu\right)$ weakly. But for every $v \in L^{p}\left(H_{T}, d t \otimes \mu\right)$ by Proposition 2.4 


$$
\begin{aligned}
& \left|\int_{0}^{T} \int_{H} v\left(B_{\epsilon}\left(u_{n}, F_{n}\right)-B_{\epsilon}(u, F)\right) d \mu d t\right| \\
& =\left|\int_{0}^{T} \int_{H} v B_{\epsilon}\left(u_{n}, F_{n}-F\right) d \mu d t+\int_{0}^{T} \int_{H} v B_{\epsilon}\left(u_{n}-u, F\right) d \mu d t\right| \\
& \leq C\|v\|_{L^{p}\left(H_{T}, d t \otimes \mu\right)}\|u\|_{L^{r}\left(H_{T}, d t \otimes \mu\right)} \\
& \quad \times\left[\left\|F_{n}-F\right\|_{1, s, T}+\left\|Q^{-1 / 2}\left(F_{n}-F\right)\right\|_{L^{s}\left(H_{T}, d t \otimes \mu\right)}\right] \\
& \quad+\int_{0}^{T} \int_{H} B_{\epsilon}(\cdot, F)^{*} v\left(u_{n}-u\right) d \mu d t \\
& \quad \rightarrow 0 \text { as } n \rightarrow \infty,
\end{aligned}
$$

where $B_{\epsilon}(\cdot, F)^{*} \in L\left(L^{p}\left(H_{T}, d t \otimes \mu\right), L^{r^{\prime}}\left(H_{T}, d t \otimes \mu\right)\right), r^{\prime}=\frac{r}{r-1}$, is the adjoint of the linear bounded operator in $L\left(L^{r}\left(H_{T}, d t \otimes \mu\right), L^{p^{\prime}}\left(H_{T}, d t \otimes \mu\right)\right)$ given by

$$
u \mapsto B_{\epsilon}(u, F)
$$

Here we have used Lemma 4.1. Hence

$$
B_{\epsilon}\left(u_{n}, F_{n}\right) \rightarrow B_{\epsilon}(u, F) \text { weakly in } L^{p^{\prime}}\left(H_{T}, d t \otimes \mu\right)
$$

By Proposition 2.4(ii), $B_{\epsilon}(u, F) \rightarrow 0$ in $L^{p^{\prime}}\left(H_{T}, d t \otimes \mu\right)$ as $\epsilon \rightarrow 0$, hence also with respect to the weak topology on $L^{p^{\prime}}\left(H_{T}, d t \otimes \mu\right)$. Since the latter is metrizable on norm balls in $L^{p^{\prime}}\left(H_{T}, d t \otimes \mu\right)$ and since $s \geq p^{\prime}$, we see that the right hand side of (2.7), weakly converges to $f$ in $L^{p^{\prime}}\left(H_{T}, d t \otimes \mu\right)$ when we let first $n \rightarrow \infty$ and then $\epsilon \rightarrow 0$. But obviously the left hand side of (2.7) is in $\mathscr{K}_{F}\left(\mathscr{D}_{T}\right)$. Therefore, we obtain that $\mathscr{K}_{F}\left(\mathscr{D}_{T}\right)$ is weakly dense in $L^{p^{\prime}}\left(H_{T}, d t \otimes \mu\right)$, since it contains $\mathscr{D}_{T}$ as a dense subset. Hence $(\mathscr{R})$ follows, since $\mathscr{K}_{F}\left(\mathscr{D}_{T}\right)$ is convex (even linear).

Remark 2.6 Let us compare our main result Theorem 2.3 with the corresponding result about uniqueness in [2] (i.e. the uniqueness part of [2, Theorem 3.1]).

We shall in fact see that they are incompatible. First of all, since we work on a separable Hilbert space $H$ and the authors of the above paper work on a separable Banach space $E$, to compare we have to assume that $E$ is a separable Hilbert space. They consider also another Hilbert space which is contained in $E=H$ and which can easily be seen to be identical to $Q^{1 / 2} H=: \mathscr{H}$ with norm $|\cdot|_{\mathscr{H}}=\left|Q^{-1 / 2} \cdot\right|_{H} \cdot \mathscr{H}$ is considered in [2] as a tangent space at every point in $H$, while in our framework the tangent space to $H$ is $H$ itself.

While condition (2.3) is also assumed in [2], instead of our condition

$$
F \in L^{s}\left([0, T] ; W^{1, s}(H ; H, \mu)\right),
$$


the authors assume that

$$
F \in L^{s}\left([0, T] ; W^{1, s}(H ; \mathscr{H}, \mu)\right),
$$

which in turn is defined to be the completion of $\mathscr{V} \mathscr{F} C_{b, T}^{1}$ with respect to the norm

$$
\left(\int_{0}^{T} \int_{H}\left(\left\|D_{\mathscr{H}} F(t, x)\right\|_{L_{2}(\mathscr{H}, \mathscr{H})}^{s}+|F(t, x)|_{\mathscr{H}}^{s}\right) \mu(d x) d t\right)^{1 / s},
$$

where $L_{2}(\mathscr{H}, \mathscr{H})$ is the space of Hilbert-Schmidt operators from $\mathscr{H}$ to $\mathscr{H}$ and analogously to (2.2) for $\varphi \in \mathscr{F} C_{b}^{1}, x \in H, D \mathscr{H} \varphi(x)$ is the unique element in $\mathscr{H}$ such that

$$
\left\langle D_{\mathscr{H}} \varphi(x), y\right\rangle_{\mathscr{H}}=\varphi^{\prime}(x)(y)=\frac{\partial \varphi}{\partial y}(x), \quad y \in \mathscr{H} .
$$

Correspondingly, for $F=\sum_{i=1}^{N} g_{i} e_{i} \in \mathscr{V} \mathscr{F} C_{b, T}^{1},(t, x) \in H_{T}$

$$
D_{\mathscr{H}} F(t, x):=\sum_{i=1}^{N}\left\langle D_{\mathscr{H}} g_{i}(t, x), \cdot\right\rangle_{\mathscr{H}} e_{i}\left(\in L_{2}(\mathscr{H}, \mathscr{H})\right) .
$$

Note that clearly $\widetilde{e_{j}}:=\lambda_{j}^{1 / 2} e_{j}, j \in \mathbb{N}$, is an orthonormal basis in $\mathscr{H}$, hence

$$
\begin{aligned}
\left\|D_{\mathscr{H}} F(t, x)\right\|_{L_{2}(\mathscr{H}, \mathscr{H})}^{2} & =\sum_{j=1}^{\infty}\left|D_{\mathscr{H}} F(t, x)\left(\widetilde{e_{j}}\right)\right|_{\mathscr{H}}^{2} \\
& =\sum_{j=1}^{\infty} \sum_{i=1}^{N} \lambda_{j}\left\langle D_{\mathscr{H}} g_{i}(t, x), e_{j}\right\rangle_{\mathscr{H}}^{2}\left\langle e_{i}, e_{i}\right\rangle \mathscr{H} \\
& \stackrel{(2.11)}{=} \sum_{i, j=1}^{N} \frac{\lambda_{j}}{\lambda_{i}}\left(\frac{\partial g_{i}}{\partial e_{j}}(t, x)\right)^{2},
\end{aligned}
$$

whereas similarly

$$
\|D F(t, x)\|_{L^{2}(H, H)}^{2}=\sum_{i, j=1}^{N}\left(\frac{\partial g_{i}}{\partial e_{j}}(t, x)\right)^{2} .
$$

Therefore, in general, neither of the function spaces in (2.9),(2.10) respectively contains the other, hence conditions (2.9), (2.10) are incompatible. A further difference to [2] is that unlike in (the uniqueness part of) [2, Theorem 3.1] we do not have to assume any exponential $\mu \otimes d t$-integrability of the Gaussian divergence of $F$, i.e. of the negative part of $\left(-D_{\mathscr{H}}^{*} F\right)$ where $D^{*}$ is the adjoint of

$$
D: W_{\mathscr{H}}^{1,2}(H, \mu) \subset L^{2}(H, \mu) \rightarrow L^{2}(H ; \mathscr{H}, \mu) .
$$

It is easy to construct examples where this exponential integrability does not hold for $F$, while $F$ satisfies all other assumptions in Theorem 2.3. 


\section{Proof of Proposition 2.4(i)}

3.1 A representation formula for the commutator

We shall use the notation

$$
\operatorname{div}_{Q} F(t, x):=\operatorname{Tr}[D F(t, x)]-\left\langle x, Q^{-1} F(t, x)\right\rangle
$$

Note that $\operatorname{Tr}[D F(t, x)]=\operatorname{div}[F(t, x)]$.

Proposition 3.1 We have

$$
\begin{aligned}
& B_{\epsilon}(u, F)(t, x)=\int_{H} \operatorname{div}_{Q} F\left(t, T_{\epsilon} x+S_{\epsilon} y\right) u\left(t, T_{\epsilon} x+S_{\epsilon} y\right) \mu(d y) \\
& \quad-\int_{H}\left[g_{\epsilon}\left(t, T_{\epsilon} x+S_{\epsilon} y,-S_{\epsilon} x+T_{\epsilon} y\right)-g_{\epsilon}(t, x, y)\right] u\left(t, T_{\epsilon} x+S_{\epsilon} y\right) \mu(d y) \\
& =: B_{\epsilon}^{1}(u, F)(t, x)-B_{\epsilon}^{2}(u, F)(t, x)
\end{aligned}
$$

where

$$
g_{\epsilon}(t, x, y):=\left\langle\frac{Q^{-1} T_{\epsilon}}{S_{\epsilon}} F(t, x), y\right\rangle .
$$

Proof Concerning the second addendum of the commutator (2.4), we have by (1.9), using a well known integration by parts formula for Gaussian measures,

$$
\begin{aligned}
\left(P_{\epsilon}\left(\left\langle F(t, \cdot), D_{x} u(t, \cdot)\right\rangle\right)(x)=\right. & \int_{H}\left\langle F(t, y), D_{y} u(t, y)\right\rangle \rho(\epsilon, x, y) \mu(d y) \\
= & -\int_{H} \operatorname{div} F(t, y) u(t, y) \rho(\epsilon, x, y) \mu(d y) \\
& -\int_{H}\left\langle F(t, y), D_{y} \rho(\epsilon, x, y)\right\rangle u(t, y) \mu(d y) \\
& +\int_{H}\left\langle Q^{-1} y, F(t, y)\right\rangle u(t, y) \rho(\epsilon, x, y) \mu(d y) .
\end{aligned}
$$

Taking into account (1.12), yields

$$
\begin{aligned}
& \left(P_{\epsilon}\left(\left\langle F(t, \cdot), D_{x} u(t, \cdot)\right\rangle\right)(x)\right. \\
& =-\int_{H} \operatorname{div}_{Q} F(t, y) u(t, y) \rho(\epsilon, x, y) \mu(d y) \\
& \quad-\int_{H}\left\langle F(t, y), Q_{\epsilon}^{-1} T_{\epsilon}\left(x-T_{\epsilon} y\right)\right\rangle u(t, y) \rho(\epsilon, x, y) \mu(d y) .
\end{aligned}
$$


Concerning the first addendum of the commutator, we have, taking into account (1.11),

$$
\begin{aligned}
\left\langle F(t, x), D_{x} P_{\epsilon} u(t, x)\right\rangle & =\int_{H}\left\langle F(t, x), D_{x} \rho(\epsilon, x, y)\right\rangle u(t, y) \mu(d y) \\
& =\int_{H}\left\langle F(t, x), Q_{\epsilon}^{-1} T_{\epsilon}\left(y-T_{\epsilon} x\right)\right\rangle u(t, y) \rho(\epsilon, x, y) \mu(d y) .
\end{aligned}
$$

So, we obtain

$$
\begin{aligned}
B_{\epsilon}(u, F)(t, x)= & \int_{H} \operatorname{div}_{Q} F(t, y) u(t, y) \rho(\epsilon, x, y) \mu(d y) \\
& +\int_{H}\left\langle F(t, x), Q_{\epsilon}^{-1} T_{\epsilon}\left(y-T_{\epsilon} x\right)\right\rangle u(t, y) \rho(\epsilon, x, y) \mu(d y) \\
& +\int_{H}\left\langle F(t, y), Q_{\epsilon}^{-1} T_{\epsilon}\left(x-T_{\epsilon} y\right)\right\rangle u(t, y) \rho(\epsilon, x, y) \mu(d y) .
\end{aligned}
$$

Since $\rho(\epsilon, x, y) \mu(d y)=N_{T_{\epsilon} x, Q_{\epsilon}}(d y)$ we can write (3.5) as

$$
\begin{aligned}
B_{\epsilon}(u, F)(t, x)= & \int_{H} \operatorname{div}_{Q} F\left(t, T_{\epsilon} x+y\right) u\left(t, T_{\epsilon} x+y\right) N_{Q_{\epsilon}}(d y) \\
& +\int_{H}\left\langle F(t, x), Q_{\epsilon}^{-1} T_{\epsilon} y\right\rangle u\left(t, T_{\epsilon} x+y\right) N_{Q_{\epsilon}}(d y) \\
& +\int_{H}\left\langle F\left(t, T_{\epsilon} x+y\right), Q_{\epsilon}^{-1} T_{\epsilon}\left(x-T_{\epsilon}\left(y+T_{\epsilon} x\right)\right)\right\rangle u\left(t, T_{\epsilon} x+y\right) N_{Q_{\epsilon}}(d y) .
\end{aligned}
$$

Since $x-T_{\epsilon}\left(y+T_{\epsilon} x\right)=x-T_{\epsilon}^{2} x-T_{\epsilon} y=S_{\epsilon}^{2} x-T_{\epsilon} y$, using the Mehler formula (1.13) we have

$$
\begin{aligned}
B_{\epsilon}(u, F)(t, x)= & \int_{H} \operatorname{div}_{Q} F\left(t, T_{\epsilon} x+S_{\epsilon} y\right) u\left(t, T_{\epsilon} x+S_{\epsilon} y\right) N_{Q}(d y) \\
& +\int_{H}\left\langle F(t, x), Q_{\epsilon}^{-1} T_{\epsilon} S_{\epsilon} y\right\rangle u\left(t, T_{\epsilon} x+S_{\epsilon} y\right) N_{Q}(d y) \\
& +\int_{H}\left\langle F\left(t, T_{\epsilon} x+S_{\epsilon} y\right), Q_{\epsilon}^{-1} T_{\epsilon} S_{\epsilon}\left(S_{\epsilon} x-T_{\epsilon} y\right)\right\rangle u\left(t, T_{\epsilon} x+S_{\epsilon} y\right) N_{Q}(d y),
\end{aligned}
$$

which coincides with (3.2).

We write now $B_{\epsilon}^{2}(u, F)(t, x)$ in a more suitable form. 
Proposition 3.2 We have

$$
\begin{aligned}
B_{\epsilon}^{2}(u, F)(t, x)=\frac{\epsilon}{2} \int_{H} \int_{0}^{1}\left[\left\langle\frac{Q^{-1} T_{\epsilon}}{S_{\epsilon}} D F\left(t, x_{\xi}\right)\left(Q^{-1} \frac{T_{\epsilon \xi}}{S_{\epsilon \xi}} y_{\xi}\right), y_{\xi}\right\rangle-\operatorname{div} G\left(t, x_{\xi}\right)\right] \\
\quad \times u\left(t, x_{1}\right) d \xi \mu(d y)-\frac{\epsilon}{2} \int_{H}^{1} \int_{0}^{1} \operatorname{div}_{Q} G\left(t, x_{\xi}\right) u\left(t, x_{1}\right) d \xi \mu(d y) \\
=: B_{\epsilon}^{2,1}(u, F)(t, x)+B_{\epsilon}^{2,2}(u, F)(t, x),
\end{aligned}
$$

where

$$
G(t, x)=Q^{-1} \frac{T_{\epsilon}}{S_{\epsilon}} \frac{T_{\epsilon \xi}}{S_{\epsilon \xi}} F(t, x)
$$

and

$$
x_{\xi}=T_{\epsilon \xi} x+S_{\epsilon \xi} y, \quad y_{\xi}=-S_{\epsilon \xi} x+T_{\epsilon \xi} y, \quad \xi \in[0,1] .
$$

Proof We start from the expression of $B_{\epsilon}^{2}(u, F)(t, x)$ given by (3.2). By (3.8) and (1.7) it follows that

$$
x=T_{\epsilon \xi} x_{\xi}-S_{\epsilon \xi} y_{\xi}, \quad y=S_{\epsilon \xi} x_{\xi}+T_{\epsilon \xi} y_{\xi} .
$$

Moreover, from (3.8)

$$
T_{\epsilon \xi} x-\frac{T_{\epsilon \xi}^{2}}{S_{\epsilon \xi}} y=-\frac{T_{\epsilon \xi}}{S_{\epsilon \xi}} y_{\xi}, \quad T_{\epsilon \xi} y+\frac{T_{\epsilon \xi}^{2}}{S_{\epsilon \xi}} x=\frac{T_{\epsilon \xi}}{S_{\epsilon \xi}} x_{\xi} .
$$

Therefore we can write

$$
B_{\epsilon}^{2}(u, F)(t, x)=\int_{H}\left[g_{\epsilon}\left(t, x_{1}, y_{1}\right)-g_{\epsilon}(t, x, y)\right] u\left(t, x_{1}\right) \mu(d y)
$$

and, taking into account that

$$
D_{\xi} x_{\xi}=-\frac{1}{2} Q^{-1} \epsilon\left(T_{\epsilon \xi} x-\frac{T_{\epsilon \xi}^{2}}{S_{\epsilon \xi}} y\right), \quad D_{\xi} y_{\xi}=\frac{1}{2} Q^{-1} \epsilon\left(-\frac{T_{\epsilon \xi}^{2}}{S_{\epsilon \xi}} x-T_{\epsilon \xi} y\right),
$$

we have

$$
\begin{aligned}
g_{\epsilon}\left(t, x_{1}, y_{1}\right)-g_{\epsilon}(t, x, y) & =\int_{0}^{1} D_{\xi} g_{\epsilon}\left(t, x_{\xi}, y_{\xi}\right) d \xi \\
& =\int_{0}^{1}\left[D_{x} g_{\epsilon}\left(t, x_{\xi}, y_{\xi}\right) D_{\xi} x_{\xi}+D_{y} g_{\epsilon}\left(t, x_{\xi}, y_{\xi}\right) D_{\xi} y_{\xi}\right] d \xi
\end{aligned}
$$




$$
\begin{aligned}
= & \int_{0}^{1} D_{x} g_{\epsilon}\left(t, x_{\xi}, y_{\xi}\right)\left(-\frac{1}{2} Q^{-1} \epsilon\left(T_{\epsilon \xi} x-\frac{T_{\epsilon \xi}^{2}}{S_{\epsilon \xi}} y\right)\right) d \xi \\
& +\int_{0}^{1} D_{y} g_{\epsilon}\left(t, x_{\xi}, y_{\xi}\right)\left(\frac{1}{2} Q^{-1} \epsilon\left(-\frac{T_{\epsilon \xi}^{2}}{S_{\epsilon \xi}} x-T_{\epsilon \xi} y\right)\right) d \xi
\end{aligned}
$$

Therefore

$$
\begin{aligned}
& B_{\epsilon}^{2}(u, F)(t, x) \\
& =\int_{H} \int_{0}^{1} D_{x} g_{\epsilon}\left(t, x_{\xi}, y_{\xi}\right)\left(-\frac{1}{2} Q^{-1} \epsilon\left(T_{\epsilon \xi} x-\frac{T_{\epsilon \xi}^{2}}{S_{\epsilon \xi}} y\right)\right) u\left(t, x_{1}\right) d \xi \mu(d y) \\
& \quad+\int_{H} \int_{0}^{1} D_{y} g_{\epsilon}\left(t, x_{\xi}, y_{\xi}\right)\left(\frac{1}{2} Q^{-1} \epsilon\left(-\frac{T_{\epsilon \xi}^{2}}{S_{\epsilon \xi}} x-T_{\epsilon \xi} y\right)\right) u\left(t, x_{1}\right) d \xi \mu(d y)
\end{aligned}
$$

But

$$
\begin{aligned}
& D_{x} g_{\epsilon}\left(t, x_{\xi}, y_{\xi}\right) z=\left\langle\frac{Q^{-1} T_{\epsilon}}{S_{\epsilon}} D F\left(t, x_{\xi}\right) z, y_{\xi}\right\rangle, \\
& D_{y} g_{\epsilon}\left(t, x_{\xi}, y_{\xi}\right) z=\left\langle\frac{Q^{-1} T_{\epsilon}}{S_{\epsilon}} F\left(t, x_{\xi}\right), z\right\rangle .
\end{aligned}
$$

Therefore from (3.12) we get

$$
\begin{aligned}
& B_{\epsilon}^{2}(F, u)(t, x) \\
& =\frac{\epsilon}{2} \int_{H} \int_{0}^{1}\left\langle\frac{Q^{-1} T_{\epsilon}}{S_{\epsilon}} D F\left(t, x_{\xi}\right)\left(-Q^{-1}\left(T_{\epsilon \xi} x-\frac{T_{\epsilon \xi}^{2}}{S_{\epsilon \xi}} y\right)\right), y_{\xi}\right\rangle u\left(t, x_{1}\right) d \xi \mu(d y) \\
& \quad+\frac{\epsilon}{2} \int_{H} \int_{0}^{1}\left\langle\frac{Q^{-1} T_{\epsilon}}{S_{\epsilon}} F\left(t, x_{\xi}\right), Q^{-1}\left(-\frac{T_{\epsilon \xi}^{2}}{S_{\epsilon \xi}} x-T_{\epsilon \xi} y\right)\right\rangle u\left(t, x_{1}\right) d \xi \mu(d y)
\end{aligned}
$$

Equivalently

$$
\begin{aligned}
B_{\epsilon}^{2}(u, F)(t, x)= & \frac{\epsilon}{2} \int_{H} \int_{0}^{1}\left\langle\frac{Q^{-1} T_{\epsilon}}{S_{\epsilon}} D F\left(t, x_{\xi}\right)\left(Q^{-1} \frac{T_{\epsilon \xi}}{S_{\epsilon \xi}} y_{\xi}\right), y_{\xi}\right) u\left(t, x_{1}\right) d \xi \mu(d y) \\
& +\frac{\epsilon}{2} \int_{H} \int_{0}^{1}\left\langle\frac{Q^{-1} T_{\epsilon}}{S_{\epsilon}} F\left(t, x_{\xi}\right), Q^{-1} \frac{T_{\epsilon \xi}}{S_{\epsilon \xi}} x_{\xi}\right\rangle u\left(t, x_{1}\right) d \xi \mu(d y),
\end{aligned}
$$


Now the conclusion follows by completing the $Q$-divergence introducing $G(t, x)$ defined by (3.7), and writing

$$
\left.\left\langle\frac{Q^{-1} T_{\epsilon}}{S_{\epsilon}} F\left(t, x_{\xi}\right), Q^{-1} \frac{T_{\epsilon \xi}}{S_{\epsilon \xi}} x_{\xi}\right)\right\rangle=-\operatorname{div}_{Q} G\left(t, x_{\xi}\right)+\operatorname{div} G\left(t, x_{\xi}\right) .
$$

\subsection{Bound of the commutator}

Let us first estimate $\left\|\mid B_{\epsilon}^{2,1}(u, F)\right\|_{L^{p^{\prime}}\left(H_{T}, d t \otimes \mu\right)}$; then we shall estimate $B_{\epsilon}^{1}(u, F)$ and $B_{\epsilon}^{2,2}(u, F)$ that are analogous.

Proposition 3.3 Let $p>2, p^{\prime}=\frac{p}{p-1}, s>p^{\prime}, \frac{1}{p^{\prime}}=\frac{1}{r}+\frac{1}{s}$. Then we have

$$
\begin{aligned}
& \left\|B_{\epsilon}^{2,1}(u, F)\right\|_{L^{p^{\prime}}\left(H_{T}, d t \otimes \mu\right)} \\
& \leq C^{\prime}\|u\|_{L^{r}\left(H_{T}, d t \otimes \mu\right)}\left(\int_{0}^{T} \int_{H}\|(D F(t, x))\|_{\mathscr{L}_{1}(H)}^{s} d t \mu(d x)\right)^{\frac{1}{s}} .
\end{aligned}
$$

Proof We recall that

$$
\begin{aligned}
B_{\epsilon}^{2,1}(u, F)(t, x)= & \frac{\epsilon}{2} \int_{H} \int_{0}^{1}\left[\left\langle\frac{Q^{-1} T_{\epsilon}}{S_{\epsilon}} D F\left(t, x_{\xi}\right)\left(Q^{-1} \frac{T_{\epsilon \xi}}{S_{\epsilon \xi}} y_{\xi}\right), y_{\xi}\right\rangle+\operatorname{div} G\left(t, x_{\xi}\right)\right] \\
& \times u\left(t, x_{1}\right) d \xi \mu(d y) \\
= & : \int_{H} \int_{0}^{1} H\left(t, x_{\xi}, y_{\xi}\right) u\left(t, x_{1}\right) d \xi \mu(d y) .
\end{aligned}
$$

Let now $v \in L^{p}\left(H_{T}, d t \otimes \mu\right)$. Then

$$
\begin{aligned}
& \left|\int_{0}^{T} \int_{H} B_{\epsilon}^{2,1}(u, F)(t, x) v(t, x) d t \mu(d x)\right| \\
& \quad \leq \int_{0}^{T} \int_{0}^{1} \int_{H} \int_{H}\left|H\left(t, x_{\xi}, y_{\xi}\right) u\left(t, x_{1}\right) v(t, x)\right| d \xi \mu(d x) d t \mu(d y) \\
& \leq \int_{0}^{1} d \xi\left(\int_{0}^{T} \int_{H} \int_{H}\left|H\left(t, x_{\xi}, y_{\xi}\right) u\left(t, x_{1}\right)\right|^{p^{\prime}} d t \mu(d x) \mu(d y)\right)^{1 / p^{\prime}}\|v\|_{L^{p}\left(H_{T}, d t \otimes \mu\right)} .
\end{aligned}
$$


By the arbitrariness of $v$ it follows that

$$
\begin{aligned}
\left\|B_{\epsilon}^{2,1}(u, F)\right\|_{L^{p^{\prime}}\left(H_{T}, d t \otimes \mu\right)} \leq & \int_{0}^{1} d \xi\left(\int_{0}^{T} \int_{H} \int_{H}\left|H\left(t, x_{\xi}, y_{\xi}\right) u\left(t, x_{1}\right)\right|^{p^{\prime}} d t\right. \\
& \times \mu(d x) \mu(d y))^{1 / p^{\prime}} .
\end{aligned}
$$

Making the change of variables (3.8) and recalling that it is invariant for $\mu \times \mu$ so that $\mu(d x) \mu(d y)=\mu\left(d x_{\xi}\right) \mu\left(d y_{\xi}\right)$, we get

$$
\begin{aligned}
& \left\|B_{\epsilon}^{2,1}(u, F)\right\|_{L^{p^{\prime}}\left(H_{T}, d t \otimes \mu\right)} \\
& \leq \int_{0}^{1} d \xi\left(\int_{0}^{T} \int_{H} \int_{H}|H(t, x, y)|^{s} \mu(d x) \mu(d y)\right)^{\frac{1}{s}}\|u\|_{L^{r}\left(H_{T}, d t \otimes \mu\right)},
\end{aligned}
$$

equivalently

$$
\begin{aligned}
& \left\|B_{\epsilon}^{2,1}(u, F)\right\|_{L^{p^{\prime}}\left(H_{T}, d t \otimes \mu\right)} \leq \frac{\epsilon}{2}\|u\|_{L^{r}\left(H_{T}, d t \otimes \mu\right)} \\
& \times \int_{0}^{1} d \xi\left(\int_{0}^{T} \int_{H} \int_{H} \mid\left\langle D F(t, x)\left(Q^{-1} \frac{T_{\epsilon \xi}}{S_{\epsilon \xi}} y\right), Q^{-1} \frac{T_{\epsilon}}{S_{\epsilon}} y\right\rangle\right. \\
& \left.-\left.\operatorname{div} G(t, x)\right|^{s} d t \mu(d x) \mu(d y)\right)^{\frac{1}{s}} .
\end{aligned}
$$

Now we estimate the integral

$$
J_{1}(t, x):=\int_{H}\left|\left\langle D F(t, x)\left(Q^{-1} \frac{T_{\epsilon \xi}}{S_{\epsilon}} y\right), Q^{-1} \frac{T_{\epsilon}}{S_{\epsilon}} y\right\rangle-\operatorname{div} G(t, x)\right|^{s} \mu(d y),
$$

applying Proposition 5.2 from Appendix 2. Setting

$$
\begin{aligned}
& L=\frac{S_{\epsilon \xi}}{T_{\epsilon \xi}} D F(t, x) Q^{-1} \frac{T_{\epsilon \xi}}{S_{\epsilon \xi}}, \\
& M=Q^{1 / 2} L Q^{1 / 2}=Q^{-1 / 2} \frac{T_{\epsilon}}{S_{\epsilon}} D F(t, x) \frac{T_{\epsilon \xi}}{S_{\epsilon \xi}} Q^{-1 / 2}
\end{aligned}
$$

and, taking into account (5.5), we obtain for $s \geq 1$

$$
\begin{aligned}
J_{1}(t, x) & \leq C_{s}\left\|\left(Q^{-1 / 2} \frac{T_{\epsilon}}{S_{\epsilon}} D F(t, x) \frac{T_{\epsilon \xi}}{S_{\epsilon \xi}} Q^{-1 / 2}\right)\right\|_{\mathscr{L}_{2}(H)}^{s} \\
& \leq C_{s}\left\|Q^{-1 / 2} \frac{T_{\epsilon}}{S_{\epsilon}}\right\|^{s}\left\|Q^{-1 / 2} \frac{T_{\epsilon \xi}}{S_{\epsilon \xi}}\right\|^{s}\|D F(t, x)\|_{\mathscr{L}_{2}(H)}^{s},
\end{aligned}
$$

having used [10, Lemma 9(d), page 1093]. 
Now we estimate $\left\|Q^{-1 / 2} \frac{T_{\epsilon}}{S_{\epsilon}}\right\|$ and $\left\|Q^{-1 / 2} \frac{T_{\epsilon \xi}}{S_{\epsilon \xi}}\right\|$.

Since

$$
\begin{aligned}
& Q^{-1 / 2} \frac{T_{\epsilon}}{S_{\epsilon}} e_{k}=\sqrt{2 \alpha_{k}} \frac{e^{-\alpha_{k} \epsilon}}{\sqrt{1-e^{-2 \alpha_{k} \epsilon}}} e_{k} \\
& Q^{-1 / 2} \frac{T_{\epsilon} \xi}{S_{\epsilon} \xi} e_{k}=\sqrt{2 \alpha_{k}} \frac{e^{-\alpha_{k} \epsilon \xi}}{\sqrt{1-e^{-2 \alpha_{k} \epsilon \xi}}} e_{k},
\end{aligned}
$$

(here $\alpha_{k}$ stands for $\frac{1}{2} \lambda_{k}^{-1}$ ) we find

$$
\left\|Q^{-1 / 2} \frac{T_{\epsilon}}{S_{\epsilon}}\right\| \leq \frac{C}{\epsilon^{1 / 2}}, \quad\left\|Q^{-1 / 2} \frac{T_{\epsilon \xi}}{S_{\epsilon \xi}}\right\| \leq \frac{C}{\epsilon^{1 / 2} \xi^{1 / 2}}
$$

So

$$
J_{1}(t, x) \leq C_{m}^{\prime} \frac{C}{\left(\epsilon \xi^{1 / 2}\right)^{s}}\|D F(t, x)\|_{\mathscr{L}_{2}(H)}^{s}
$$

Now by (3.17) we obtain

$$
\begin{aligned}
& \left\|B_{\epsilon}^{2,1}(u, F)\right\|_{L^{p^{\prime}}\left(H_{T}, d t \otimes \mu\right)} \\
& \leq C^{\prime} \frac{1}{2}\|u\|_{L^{r}\left(H_{T}, d t \otimes \mu\right)} \int_{0}^{1} \xi^{1 / 2} d \xi\left(\int_{0}^{T} \int_{H}\|D F(t, x)\|_{\mathscr{L}_{2}(H)}^{s} d t \mu(d x)\right)^{\frac{1}{s}} \\
& =C^{\prime}\|u\|_{L^{r}\left(H_{T}, d t \otimes \mu\right)}\left(\int_{0}^{T} \int_{H}\|D F(t, x)\|_{\mathscr{L}_{2}(H)}^{s} d t \mu(d x)\right)^{\frac{1}{s}} .
\end{aligned}
$$

\section{Proposition 3.4 We have}

$$
\begin{aligned}
& \left\|B_{\epsilon}^{1}(u, F)\right\|_{L^{p^{\prime}}\left(H_{T}, d t \otimes \mu\right)}+\left\|B_{\epsilon}^{2,2}(u, F)\right\|_{L^{p^{\prime}}\left(H_{T}, d t \otimes \mu\right)} \\
& \leq C\|u\|_{L^{r}\left(H_{T}, d t \otimes \mu\right)}\left(\int_{0}^{T} \int_{H}\left(|D F(t, x)|_{\mathscr{L}_{2}(H)}^{s}+\left|Q^{-1 / 2} F(t, x)\right|^{s}\right) d t \mu(d x)\right)^{\frac{1}{s}} .
\end{aligned}
$$

Proof Let us first deal with $B_{\epsilon}^{2,2}(u, F)$ which is more difficult. Recall that

$$
B_{\epsilon}^{2,2}(u, F)(t, x)=\frac{\epsilon}{2} \int_{H} \int_{0}^{1} \operatorname{div} Q\left[Q^{-1} \frac{T_{\epsilon}}{S_{\epsilon}} \frac{T_{\epsilon \xi}}{S_{\epsilon \xi}} F\left(t, x_{\xi}\right)\right] u\left(t, x_{1}\right) d \xi \mu(d y) .
$$


Proceeding as in the proof of (3.17) and using again the change of variables (3.8), we find

$$
\begin{aligned}
& \left\|B_{\epsilon}^{2,2}(u, F)\right\|_{L^{p^{\prime}\left(H_{T}, d t \otimes \mu\right)}} \leq \frac{\epsilon}{2}\|u\|_{L^{r}\left(H_{T}, d t \otimes \mu\right)} \\
& \times \int_{0}^{1} d \xi\left(\int_{0}^{T} \int_{H} \int_{H}\left|\operatorname{div}_{Q}\left[Q^{-1} \frac{T_{\epsilon}}{S_{\epsilon}} \frac{T_{\epsilon \xi}}{S_{\epsilon \xi}} F(t, x)\right]\right|^{s} d t \mu(d x) \mu(d y)\right)^{\frac{1}{s}} .
\end{aligned}
$$

Now, taking into account Lemma 5.3 of Appendix 2, we find

$$
\begin{aligned}
\left\|B_{\epsilon}^{2,2}(u, F)\right\|_{L^{p^{\prime}}\left(H_{T}, d t \otimes \mu\right)} \leq \frac{\epsilon}{2} & C_{S}\|u\|_{L^{r}\left(H_{T}, d t \otimes \mu\right)} \\
& \times \int_{0}^{1} d \xi\left(\int _ { 0 } ^ { T } \int _ { H } \int _ { H } \left(\left|Q^{-1} \frac{T_{\epsilon}}{S_{\epsilon}} \frac{T_{\epsilon \xi}}{S_{\epsilon \xi}} D F(t, x)\right|_{\mathscr{L}_{2}(H)}^{s}\right.\right. \\
& \left.\left.+\left|Q^{-1 / 2} Q^{-1} \frac{T_{\epsilon}}{S_{\epsilon}} \frac{T_{\epsilon \xi}}{S_{\epsilon \xi}} F(t, x)\right|^{s}\right) d t \mu(d x) \mu(d y)\right)^{\frac{1}{s}} .
\end{aligned}
$$

Now by (3.20) we obtain (3.22) for $\left\|B_{\epsilon}^{2,2}(u, F)\right\|_{L^{p^{\prime}}\left(H_{T}, d t \otimes \mu\right)}$. Similarly, from the definition of $B_{\epsilon}^{1}(u, F)$,

$$
\begin{aligned}
& \left\|B_{\epsilon}^{1}(u, F)\right\|_{L^{p^{\prime}}\left(H_{T}, d t \otimes \mu\right)} \\
& \quad \leq C\|u\|_{L^{r}\left(H_{T}, d t \otimes \mu\right)}\left(\int_{0}^{T} \int_{H}\left(\left|\operatorname{div}_{Q} F(t, x)\right|_{\mathscr{L}_{2}(H)}^{s} d t \mu(d x)\right)^{\frac{1}{s}} .\right.
\end{aligned}
$$

\section{Appendix 1}

Let $p>1$ be given. Denote by $W_{Q}^{1, p}$ the space of ( $\mu$-equivalence classes of) Borel measurable vector fields $G: H \rightarrow D\left(Q^{-1 / 2}\right)$, having Fréchet differential $D G(x) \in$ $L_{2}(H, H)$ for $\mu$-a.e. $x \in H$, such that

$$
\|G\|_{W_{Q}^{1, p}}^{p}:=\int_{H}\left(\left|Q^{-1 / 2} G(x)\right|^{p}+\|D G(x)\|_{L_{2}(H, H)}^{p}\right) \mu(d x)<\infty .
$$

The space $W_{Q}^{1, p}$ is a separable Banach space with the norm $\|G\|_{W_{Q}^{1, p}}$. Consider the space $L^{p}\left(0, T ; W_{Q}^{1, p}\right)$ with the norm $\|F\|_{L^{p}\left(W_{Q}^{1, p}\right)}$ defined as 


$$
\begin{aligned}
\|F\|_{L^{p}\left(W_{Q}^{1, p}\right)}^{p} & =\int_{0}^{T}\|F(t, \cdot)\|_{W_{Q}^{1, p}}^{p} d t \\
& =\int_{0}^{T} \int_{H}\left(\left|Q^{-1 / 2} F(t, x)\right|^{p}+\|D F(t, x)\|_{L_{2}(H, H)}^{p}\right) \mu(d x) d t .
\end{aligned}
$$

Lemma 4.1 Denote by $\mathscr{V}_{p}$ the family of all functions $F_{n} \in L^{p}\left(0, T ; W_{Q}^{1, p}\right)$ of the form

$$
F_{n}(t, x)=\sum_{i=1}^{n} \varphi_{i}^{n}\left(t,\left\langle x, e_{1}\right\rangle, \ldots,\left\langle x, e_{n}\right\rangle\right) e_{i}
$$

with $\varphi_{i}^{n} \in C_{0}^{1}\left([0, T] \times \mathbb{R}^{n}, \mathbb{R}\right)$. Then $\mathscr{V}_{p}$ is dense in $L^{p}\left(0, T ; W_{Q}^{1, p}\right)$.

Proof We proceed by a sequence of reductions of the problem: from general elements $F$ of $L^{p}\left(0, T ; W_{Q}^{1, p}\right)$ to piece-wise constant (in time) functions; then finitely based; then also with values in finite dimensional spaces; and finally smooth.

Step 1 Let $\mathscr{V}_{p}^{1}$ be the family of all piece-wise constant functions $F:[0, T] \rightarrow W_{Q}^{1, p}$, namely of the form

$$
F(t, \cdot)=\sum_{i=1}^{k-1} F_{i} 1_{\left[t_{i}, t_{i+1}\right]}(t)
$$

where $0 \leq t_{1} \leq \cdots \leq t_{k} \leq T$, and $F_{i} \in W_{Q}^{1, p}$. It is a known fact that $\mathscr{V}_{p}^{1}$ is dense in $L^{p}\left(0, T ; W_{Q}^{1, p}\right)$. Thus, to prove the lemma, it is sufficient to prove that any element $F \in \mathscr{V}_{p}^{1}$ can be approximated by a sequence $\left\{F_{n}\right\} \subset \mathscr{V}_{p}$, in the sense of $\lim _{n \rightarrow \infty}\left\|F_{n}-F\right\|_{L^{p}\left(W_{Q}^{1, p}\right)}^{p}=0$.

Step 2 Any $G \in W_{Q}^{1, p}$ is the limit in $\|\cdot\|_{W_{Q}^{1, p}}$ of a sequence $\left\{G_{n}\right\} \subset W_{Q}^{1, p}$ having the following property: $G_{n}(x)=G_{n}\left(\pi_{n} x\right)$ (namely they are finitely based), where $\pi_{n} x=\sum_{i=1}^{n}\left\langle x, e_{i}\right\rangle e_{i}$. Indeed, define

$$
\begin{aligned}
& G_{n}(x):=\int_{H} G\left(\pi_{n} x+\left(1-\pi_{n}\right) y\right) \mu(d y) \\
& H_{n}(x):=Q^{-1 / 2} G_{n}(x)=\int_{H} Q^{-1 / 2} G\left(\pi_{n} x+\left(1-\pi_{n}\right) y\right) \mu(d y)
\end{aligned}
$$

In [3, Corollary 3.5.2] it is proved that $H_{n} \rightarrow Q^{-1 / 2} G$ in $L^{2}(H, \mu)$, which is the first part of the property $\left\|G_{n}-G\right\|_{W_{Q}^{1, p}} \rightarrow 0$. The second one is proved in [3, Proposition 5.4.5]. 
Step 3 Any $G \in W_{Q}^{1, p}$ is the limit in $\|\cdot\|_{W_{Q}^{1, p}}$ of a sequence $\left\{G_{n}\right\} \subset W_{Q}^{1, p}$ having the following property: $G_{n}(x)=\pi_{n} G_{n}\left(\pi_{n} x\right)$ (namely they are finitely based and have values in a finite dimensional space). The proof (using Step 2) is elementary.

From these facts it follows that any element $F \in \mathscr{V}_{p}^{1}, F(t, \cdot)=\sum_{i=1}^{k-1} F_{i} 1_{\left[t_{i}, t_{i+1}\right]}(t)$, can be approximated in $L^{p}\left(W_{Q}^{1, p}\right)$-norm by $F_{n} \in \mathscr{V}_{p}^{1}$ of the form

$$
F_{n}(t, \cdot)=\sum_{i=1}^{k-1} F_{i}^{n} 1_{\left[t_{i}, t_{i+1}\right]}(t)
$$

where each $F_{i}^{n}$ has the property $F_{i}^{n}(x)=\pi_{n} F_{i}^{n}\left(\pi_{n} x\right)$ and $F_{i}^{n}$ converges to $F_{i}$ in $W_{Q}^{1, p}$.

In other words, we have proved that any $F \in \mathscr{V}_{p}^{1}$ is the limit in $L^{p}\left([0, T] ; W_{Q}^{1, p}\right)$ norm of a sequence $F_{n}$ of the form

$$
F_{n}(t, x)=\sum_{i=1}^{n} \varphi_{i}^{n}\left(t,\left\langle x, e_{1}\right\rangle, \ldots,\left\langle x, e_{n}\right\rangle\right) e_{i}
$$

where $\varphi_{i}^{n}$ are piece-wise constant in $t$ and of class $W^{1, p}\left(\mathbb{R}^{n}, \gamma_{n}\right)$ in space, where $\gamma_{n}$ is the centered symmetric Gaussian measure on $\mathbb{R}^{n}\left(\gamma_{n}\right.$ is equivalent to the Gaussian measure on $\mathbb{R}^{n}$ corresponding to the projection of $\mu$ by $\pi_{n}$, and the spaces $W^{1, p}$ coincide).

Step 4 Any element $\varphi_{i}^{n}$ of class $L^{p}\left(0, T ; W^{1, p}\left(\mathbb{R}^{n}, \gamma_{n}\right)\right)$ is limit, in such topology, of $C_{0}^{1}\left([0, T] \times \mathbb{R}^{n}, \mathbb{R}\right)$-functions. The proof is complete.

\section{Appendix 2}

\subsection{Computation of some integrals}

Let $\mu=N_{Q}$ and assume that the sequence $\left(\lambda_{k}\right)$ of eigenvalues of $Q$ be nonincreasing. For any $L \in \mathscr{L}(H)$ we denote by $L_{\sigma}$ the symmetric part of $L$, namely $L_{\sigma}=\frac{1}{2}(L+$ $\left.L^{*}\right)$. Notice that $\langle L x, x\rangle=\left\langle L_{\sigma} x, x\right\rangle$ for all $x \in H$.

Lemma 5.1 Assume that $L \in L(H)$ is compact. Then there is $\epsilon_{0}>0$ such that

$$
\begin{aligned}
\int_{H} e^{-\epsilon\langle L x, x\rangle} N_{Q}(d x) & =\int_{H} e^{-\epsilon\left\langle L_{\sigma} x, x\right\rangle} N_{Q}(d x) \\
& =\left[\operatorname{det}\left(1+2 \epsilon Q^{1 / 2} L_{\sigma} Q^{1 / 2}\right)\right]^{-1 / 2}, \text { if } \epsilon\left\langle\epsilon_{0} .\right.
\end{aligned}
$$

( $\epsilon_{0}$ is determined by the condition $1+2 \epsilon_{0} \mu>0$ where $\mu$ are eigenvalues of $\left.Q^{1 / 2} L_{\sigma} Q^{1 / 2}\right)$ ) 
Proof Set $M=Q^{1 / 2} L_{\sigma} Q^{1 / 2}, M$ is obviously compact. Let $\left(f_{k}\right)$ be an orthonormal basis of eigenvectors of $M$ and $\left(\beta_{k}\right)$ the corresponding sequence of eigenvalues. Then we have

$$
\left\langle L_{\sigma} x, x\right\rangle=\left\langle M Q^{-1 / 2} x, Q^{-1 / 2} x\right\rangle=\sum_{k=1}^{\infty} \beta_{k}\left|\left\langle Q^{-1 / 2} x, f_{k}\right\rangle\right|^{2},
$$

so that

$$
\int_{H} e^{-\epsilon\left\langle L_{\sigma} x, x\right\rangle} N_{Q}(d x)=\int_{H} e^{-\epsilon \sum_{k=1}^{\infty} \beta_{k}\left|\left\langle Q^{-1 / 2} x, f_{k}\right\rangle\right|^{2}} N_{Q}(d x)
$$

Since $\left(f_{k}\right)$ is an orthogonal system, the sequence of real random variables $x \rightarrow$ $\left\langle Q^{-1 / 2} x, f_{k}\right\rangle, k \in \mathbb{N}$ (whose law is $N_{1}$ ) are independent (We have set $\left\langle Q^{-1 / 2} x, f_{k}\right\rangle=$ $\sum_{j=1}^{\infty} \lambda_{j}^{-1 / 2}\left\langle x, e_{j}\right\rangle\left\langle f_{k}, e_{j}\right\rangle$. One checks easily that the series is convergent in $\left.L^{2}(\Omega)\right)$. Consequently

$$
\begin{aligned}
\int_{H} e^{-\epsilon\left\langle L_{\sigma} x, x\right\rangle} N_{Q}(d x) & =\prod_{k=1}^{\infty} \int_{H} e^{-\epsilon \beta_{k}\left|\left\langle Q^{-1 / 2} x, f_{k}\right\rangle\right|^{2}} N_{Q}(d x) \\
& =\prod_{k=1}^{\infty}\left(1+2 \epsilon \beta_{k}\right)^{-1 / 2} \\
& =\left[\operatorname{det}\left(1+2 \epsilon Q^{1 / 2} L_{\sigma} Q^{1 / 2}\right)\right]^{-1 / 2}
\end{aligned}
$$

which proves (5.1).

Set now

$$
\begin{aligned}
S(\epsilon) & =\int_{H} e^{-\epsilon\left(\left\langle L_{\sigma} x, x\right\rangle-\operatorname{Tr}\left[Q^{1 / 2} L_{\sigma} Q^{1 / 2}\right]\right.} N_{Q}(d x) \\
& =\left[\operatorname{det}\left(1+2 \epsilon Q^{1 / 2} L_{\sigma} Q^{1 / 2}\right)\right]^{-1 / 2} e^{\epsilon \operatorname{Tr}}\left[Q^{1 / 2} L_{\sigma} Q^{1 / 2}\right]
\end{aligned}
$$

Notice that $S(0)=1$. Then for any $m \in \mathbb{N}$ we have

$$
\int_{H}\left(\left\langle L_{\sigma} x, x\right\rangle-\operatorname{Tr}\left[Q^{1 / 2} L_{\sigma} Q^{1 / 2}\right]\right)^{m} N_{Q}(d x)=(-1)^{m} S^{(m)}(0) .
$$

Proposition 5.2 For any $s \geq 1$ there is $C_{s}>0$ such that.

$$
\int_{H}\left|\langle L x, x\rangle-\operatorname{Tr}\left[Q^{1 / 2} L Q^{1 / 2}\right]\right|^{s} N_{Q}(d x) \leq C_{S}\left\|Q^{1 / 2} L Q^{1 / 2}\right\|_{\mathscr{L}_{2}(H)}^{s}
$$


Proof We first show that

$$
\int_{H}\left|\left\langle L_{\sigma} x, x\right\rangle-\operatorname{Tr}\left[Q^{1 / 2} L_{\sigma} Q^{1 / 2}\right]\right|^{s} N_{Q}(d x) \leq C_{s}\left\|Q^{1 / 2} L_{\sigma} Q^{1 / 2}\right\|_{\mathscr{L}_{2}(H)}
$$

It is enough to prove (5.6) for $s \in \mathbb{N}$, then the general case will follow from the RiezThorin interpolation theorem. In the following we take for simplicity $s=m$ even (If $n$ is odd we use Hölder's inequality and reduce the integral in (5.6) to an integral with $2 n$ replacing $n$ ).

Setting $M=Q^{1 / 2} L_{\sigma} Q^{1 / 2}$ and taking into account that

$$
\frac{d}{d \epsilon} \operatorname{det}(1+2 \epsilon M)=2 \operatorname{det}(1+2 \epsilon M) \operatorname{Tr}\left[M(1+2 \epsilon M)^{-1}\right],
$$

we have

$$
S^{\prime}(\epsilon)=\operatorname{Tr}\left[M-M(1+2 \epsilon M)^{-1}\right] S(\epsilon)=2 \epsilon \operatorname{Tr}\left[M^{2}(1+2 \epsilon M)^{-1}\right] S(\epsilon) .
$$

In particular, $S^{\prime}(0)=0$. Now set

$$
F(\epsilon)=\log S(\epsilon) \text {. }
$$

Then

$$
F^{\prime}(\epsilon)=2 \epsilon \operatorname{Tr}\left[M^{2}(1+2 \epsilon M)^{-1}\right]
$$

and

$$
F^{(n)}(\epsilon)=(-1)^{n+1} 2^{n-1}(n-1) ! \operatorname{Tr}\left[M^{n}(1+2 \epsilon M)^{-n}\right], \quad n \geq 2 .
$$

Therefore

$$
F^{\prime}(0)=0, \quad F^{(n)}(0)=(-1)^{n+1} 2^{n-1}(n-1) ! \operatorname{Tr}\left[M^{n}\right]=: k_{n} \operatorname{Tr}\left[M^{n}\right], \quad n \geq 2 .
$$

Now $S(\epsilon)=e^{F(\epsilon)}$. We claim that there exists $C_{n}>0$ such that

$$
S^{(n)}(0) \leq C_{n}\|M\|_{\mathscr{L}_{2}(H)}^{n} .
$$

The claim follows by recurrence noting that, given $k \in \mathbb{N}$, we have ${ }^{1}$

$$
\left|\operatorname{Tr}\left[M^{k}\right]\right| \leq\left\|M^{k}\right\| \mathscr{L}_{1}(H) \leq 2^{k-1}\|M\|_{\mathscr{L}_{2}(H)}^{k},
$$

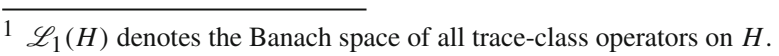


where the first inequality follows by [10, Corollary 8,page 1093] and the second one by a generalization of [10, Lemma 9(c),page 1093]. More precisely, by [10, Lemma 9(c),page 1093] it follows that

$$
\left\|M^{2}\right\| \mathscr{L}_{1(H)} \leq 2\|M\|_{\mathscr{L}_{2}(H)}^{2}
$$

and also

$$
\left\|M^{3}\right\|_{\mathscr{L}_{1}(H)} \leq 2\|M\|_{\mathscr{L}_{2}(H)}\left\|M^{2}\right\|_{\mathscr{L}_{2}(H)} \leq 2\|M\|_{\mathscr{L}_{2}(H)}\left\|M^{2}\right\|_{\mathscr{L}_{1}(H)} \leq 4\|M\|_{\mathscr{L}_{2}(H)}^{3} .
$$

Iterating this argument, (5.8) follows.

Now from the claim we obtain (5.6). To conclude the proof it is enough to note that

$$
\begin{aligned}
\left\langle L_{\sigma} x, x\right\rangle & =\langle L x, x\rangle, \quad \forall x \in H, \\
\operatorname{Tr}\left[Q^{1 / 2} L_{\sigma} Q^{1 / 2}\right] & =\operatorname{Tr}\left[Q^{1 / 2} L Q^{1 / 2}\right]
\end{aligned}
$$

and

$$
\begin{aligned}
\left\|Q^{1 / 2} L_{\sigma} Q^{1 / 2}\right\|_{\mathscr{L}_{2}(H)} & \leq \frac{1}{2}\left(\left\|Q^{1 / 2} L Q^{1 / 2}\right\|_{\mathscr{L}_{2}(H)}+\left\|Q^{1 / 2} L^{*} Q^{1 / 2}\right\|_{\mathscr{L}_{2}(H)}\right) \\
& \leq\left\|Q^{1 / 2} L Q^{1 / 2}\right\|_{\mathscr{L}_{2}(H)} .
\end{aligned}
$$

\subsection{An estimate for Gaussian divergences}

In the next lemma, $G$ is a vector field of the form

$$
G(x)=\sum_{i=1}^{n} \varphi_{i}\left(\left\langle x, e_{1}\right\rangle, \ldots,\left\langle x, e_{n}\right\rangle\right) e_{i}
$$

with $\varphi_{i} \in C_{0}^{1}\left(\mathbb{R}^{n}, \mathbb{R}\right)$, where $\left\{e_{i}\right\}$ is a c.o.s. of $H$ of eigenvectors of $Q$ (By $C_{0}^{1}\left(\mathbb{R}^{n}, \mathbb{R}\right)$ we mean the set all mappings $\mathbb{R}^{n} \times \mathbb{R} \rightarrow \mathbb{R}$ which are of compactly supported and which are continuous together their first derivatives). For them we may define $\operatorname{div}_{Q} G(y)=\operatorname{Tr}(D G(y))-\left\langle y, Q^{-1} G(y)\right\rangle$.

Lemma 5.3 For every $p>1$ there is a constant $C_{p}>0$ such that

$$
\int_{H}\left|\operatorname{div}_{Q} G(y)\right|^{p} \mu(d y) \leq C_{p} \int_{H}\left(\|D G(y)\|_{\mathscr{L}_{2}(H)}^{p}+\left|Q^{-1 / 2} G(y)\right|^{p}\right) \mu(d y)
$$

for every vector field $G$ as above. 
Proof The following result is classical in Malliavin calculus (see for instance [3, Proposition 5.8.8]): for every $p>1$ there is a constant $C_{p}>0$ such that for every $n \in \mathbb{N}$, if $\gamma_{n}$ denotes the symmetric centered Gaussian measure in $\mathbb{R}^{n}$, then

$$
\begin{aligned}
& \int_{\mathbb{R}^{n}}|\operatorname{Tr}(D F(\widetilde{x}))-\langle\widetilde{x}, F(\widetilde{x})\rangle|^{p} \gamma_{n}(d \widetilde{x}) \\
& \quad \leq C_{p} \int_{\mathbb{R}^{n}}\left(\|D F(\widetilde{x})\|_{\mathscr{L}_{2}\left(\mathbb{R}^{n}\right)}^{p}+|F(\widetilde{x})|_{\mathbb{R}^{n}}^{p}\right) \gamma_{n}(d \widetilde{x})
\end{aligned}
$$

for all smooth compact support vector fields $F: \mathbb{R}^{n} \rightarrow \mathbb{R}^{n}$.

Set $H_{n}=\pi_{n}(H), \pi_{n} x=\sum_{i=1}^{n}\left\langle x, e_{i}\right\rangle e_{i}$ and let $J: H_{n} \rightarrow \mathbb{R}^{n}$ be the natural isomorphism. The operators $Q, Q^{1 / 2}, Q^{-1 / 2}$ work as operators on $H_{n}$, hence they define corresponding operators $Q_{n}, Q_{n}^{1 / 2}, Q_{n}^{-1 / 2}$ in $\mathbb{R}^{n}$.

Given $G$ as above, consider the vector field $F: \mathbb{R}^{n} \rightarrow \mathbb{R}^{n}$ defined as

$$
F(\widetilde{x}):=Q_{n}^{-1 / 2} J G\left(J^{-1} Q_{n}^{1 / 2} \widetilde{x}\right) .
$$

With little abuse of notations, it is simply the map $F(x):=Q^{-1 / 2} G\left(Q^{1 / 2} x\right)$. We have

$$
\begin{aligned}
D F(\widetilde{x})= & J(D G)\left(J^{-1} Q_{n}^{1 / 2} \tilde{x}\right) \\
\operatorname{Tr}(D F(\widetilde{x}))-\langle\widetilde{x}, F(\widetilde{x})\rangle= & \operatorname{Tr}\left(J(D G)\left(J^{-1} Q_{n}^{1 / 2} \tilde{x}\right)\right) \\
& -\left\langle\widetilde{x}, Q_{n}^{-1 / 2} J G\left(J^{-1} Q_{n}^{1 / 2} \tilde{x}\right)\right\rangle \\
= & \left.\operatorname{div}_{Q} G(y)\right|_{y=J^{-1} Q_{n}^{1 / 2} \tilde{x}}
\end{aligned}
$$

hence we have

$$
\begin{aligned}
& \int_{\mathbb{R}^{n}}\left|\operatorname{div}_{Q} G(y)\right|_{y=\left.J^{-1} Q_{n}^{1 / 2} \widetilde{x}\right|^{p} \gamma_{n}(d \widetilde{x})} \\
& \quad \leq C_{p} \int_{\mathbb{R}^{n}}\left(\left\|J(D G)\left(J^{-1} Q_{n}^{1 / 2} \tilde{x}\right)\right\|_{\mathscr{L}_{2}\left(\mathbb{R}^{n}\right)}^{p}+\left|Q_{n}^{-1 / 2} J G\left(J^{-1} Q_{n}^{1 / 2} \widetilde{x}\right)\right|_{\mathbb{R}^{n}}^{p}\right) \gamma_{n}(d \widetilde{x}) .
\end{aligned}
$$

If we denote by $\mu_{n}$ the image measure, on $H_{n}$, of $\gamma_{n}$ under the transformation $\widetilde{x} \mapsto$ $y=J^{-1} Q_{n}^{1 / 2} \tilde{x}$, we have proved

$$
\begin{aligned}
& \int_{H}\left|\operatorname{div}_{Q} G(y)\right|^{p} \mu_{n}(d y) \\
& \quad \leq C_{p} \int_{H}\left(\|J(D G)(y)\|_{\mathscr{L}_{2}\left(\mathbb{R}^{n}\right)}^{p}+\left|Q_{n}^{-1 / 2} J G(y)\right|_{\mathbb{R}^{n}}^{p}\right) \mu_{n}(d y) .
\end{aligned}
$$

It is now easy to realize that this is the claim of the lemma, taking into account the special form of $G$. The proof is complete. 


\section{References}

1. Ambrosio, L.: Transport equation and Cauchy problem for BV vector fields. Invent. Math. 158(2), 227-260 (2004)

2. Ambrosio, L., Figalli, A.: On flows associated to Sobolev vector fields in Wiener spaces: an approach à la Di Perna - Lions. J. Funct. Anal. 256, 179-214 (2009)

3. Bogachev, V.: Gaussian Measures. American Mathematical Society, Providence (1998)

4. Bogachev, V., Da Prato, G., Röckner, M.: Existence and uniqueness of solutions for Fokker-Planck equations on Hilbert spaces. J. Evol. Equ. 10(3), 487-509 (2010)

5. Bogachev, V., Da Prato, G., Röckner, M.: Uniqueness for solutions of Fokker-Planck equations on infinite dimensional spaces. Commun. Partial Differ. Equ. 36, 925-939 (2011)

6. Bogachev, V., Da Prato, G., Röckner, M., Shaposhnikov, V.: An Analytic Approach to Infinite Dimensional FokkerPlanckKolmogorov Equations, preprint.

7. Da Prato, G.: Kolmogorov Equations for Stochastic PDEs. Birkhäuser, Basel (2004)

8. Da Prato, G.: Introduction to Stochastic Analysis and Malliavin Calculus. Edizioni della Normale, Pisa (2011)

9. Di Perna, R.J., Lions, P.L.: Ordinary differential equations, transport theory and Sobolev spaces. Invent. Math. 98, 511-547 (1989)

10. Dunford, N., Schwartz, J.T.: Linear Operators, Part II. Interscience, New York (1963)

11. Fang, S., Luo, D.: Transport equations and quasi-invariant flows on the Wiener space. Bull. Sci. Math. 134, 295-328 (2010)

12. Kolesnikov, A., Röckner, M.: On continuity equations in infinite dimensions with non-Gaussian reference measure. J. Funct. Anal. 266(7), 4490-4537 (2014)

13. Malliavin, P.: Stochastic Analysis. Springer, New York (1997)

14. Nualart, D.: The Malliavin Calculus and Related Topic. Springer, Berlin (1995) 